\title{
IoT Embedded System for Data Acquisition using MQTT Protocol
}

\author{
Vitor V. de Moura \\ Computer Science Department, \\ Federal Institute of Ceará, Brazil
}

\author{
Renata I. S. Pereira \\ Electrical Engineering Department, \\ Federal University of Ceará, Brazil
}

\author{
Sandro C. S. Jucá \\ Computer Science Department, \\ Federal Institute of Ceará, Brazil
}

\begin{abstract}
The proposed project aims to develop a data acquisition system using the Internet of things concept. The system monitors the environment in which a solar photovoltaic module is located and sends the generated voltage, ambient temperature and light incidence data to a cloud server through the Message Queuing Telemetry Transport (MQTT) communication protocol. The MQTT message is listened by a program running on the cloud server and the data received is stored on the same cloud server and can be visualized by any allowed remote user.
\end{abstract}

\section{General Terms}

Computing, Embedded systems, Online monitoring

\section{Keywords}

Data acquisition, IoT, MQTT

\section{INTRODUCTION}

Nowadays, the new global trend is the search for diversification of the electric energy matrix, mainly through renewable energy sources. Brazil has a great advantage over other countries, due to the location close to the equator, which guarantees twelve hours of sunshine per day throughout the year. In addition to the great extension of the coast, which favors the source of wind energy.

Solar energy is one of the alternative forms of generation that have more focus in developed countries because of the cheapening of solar systems and the valuation of environmental and social costs, solar photovoltaic systems tends to become economically competitive in short term [1].

Data acquisition systems use sensors to obtain information about the environment in which they are inserted and have great importance to analyze several factors that influence the energy generation [2]. It is common in acquisition systems to store the values of the readings in a database to later treat and analyze them [3]. However, with the spread of the Internet of things (IoT) concepts [4], data acquisition and monitoring systems are increasingly using remote storage on cloud servers [5, 6].

The proposed project aims to develop a data acquisition system that has Internet connection, so that sensor readings are sent via $\mathrm{WiFi}$ using the Message Queuing Telemetry Transport (MQTT) protocol [7], that uses low bandwidth.

\section{RELATED WORK}

The use of monitoring systems is common, however, with the advancement of communication technologies and protocols, these systems have gained more interaction and stability [8]. There are several papers that address the concept of the Internet of Things and data acquisition, such as the article described by Dornelas that proposes the monitoring of domestic water consumption using concepts of Internet of things [9]. In this model, Dornelas uses the open-source meta-platform KNoT, which aims to facilitate communication between hardware devices (sensors, microcontroller and microcomputer) through software, libraries and protocols.

Another work that addresses data acquisition is proposed by [10]. The system monitors energy consumption in real time in a noninvasive way using the Arduino microcontroller [11]. This developed device also performs non-invasive electrical current monitoring using a current sensor (ammeter pliers) and sends the data to the Internet using Arduino's Ethernet shield.

Using concepts of IoT allied to wireless sensor networks, the work proposed by [12] aims to develop a system of real-time monitoring sound noises in urban environments. The project contains a network of wireless sensors capable of communicating using open transmission protocols. As a form of wireless communication, this model sends data from the sensor network using a transceiver module (SIM800L) for GPRS (General Packet Radio Service) communication.

Table 1 shows a comparison table between three projects mentioned above and the proposed project.

\section{MATERIALS}

In this section, the components and technologies selected for the development of this project are described.

\subsection{ESP8266 NodeMCU Dev Kit V1.0}

The ESP8266 NodeMCU [13] microcontroller is a development board that has serial converter, voltage regulator, analog pin, WiFi modem and several other input and output pins. A representation of the version of the NodeMCU used can be seen in Figure 1. also containing the pin map of the microcontroller. The choice of 
Table 1. Comparison between the related works and the proposed project

\begin{tabular}{|c|c|c|c|}
\hline$[\mathbf{9}]$ & Microcontroller & Data Transfer & Protocol Used \\
\hline$[\mathbf{1 0}]$ & Arduino UNO & Radio & KnoT Protocol \\
\hline$[\mathbf{1 2}]$ & $\begin{array}{c}\text { Microcontroller } \\
\text { with ARM cortex }\end{array}$ & Mobile Network & $\begin{array}{c}\text { SPI, } \\
\text { TCP/UDP }\end{array}$ \\
\hline $\begin{array}{c}\text { IEEE 802.15.4, } \\
\text { Proper IPv6 and GPRS/GSM } \\
\text { for mobile connection }\end{array}$ \\
project & $\begin{array}{c}\text { NodeMCU } \\
\text { DevKit V1.0 } \\
\text { with ESP8266 } \\
\text { module }\end{array}$ & WiFi Network & $\begin{array}{c}\text { MQTT Communication } \\
\text { protocol }\end{array}$ \\
\hline
\end{tabular}

ESP8266 NodeMCU was made due to the presence of an analog pin that is used to read the values of the sensors, for the ease in programming and internet connection via $\mathrm{WiFi}$.

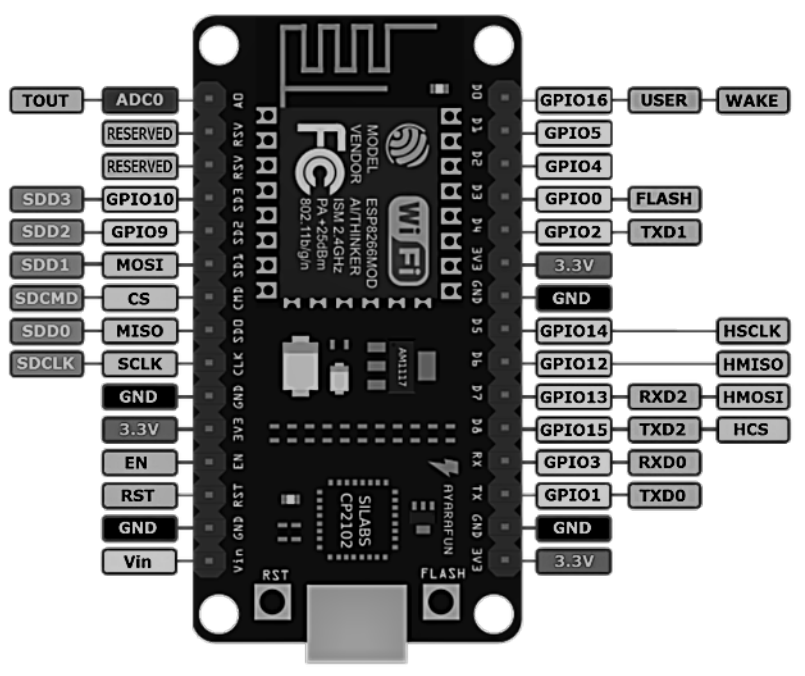

Fig. 1. NodeMCU V1.0.

\subsection{Project sensors and components}

The physical configuration of the monitoring system consists of a prototype with the objective of testing the MQTT communication and validating the proposal. The sensors used in the design are: temperature sensor (LM35), a light sensitive resistor (LDR) and a solar photovoltaic module of 6V. Considering that the ESP8266 NodeMCU has only one input with AD (analog/digital) converter, it was necessary to use a multiplexer. The integrated circuit CD74HC4051E [14] was selected. The sensors used can be seen in Figure 2

\subsection{MQTT comunication protocol}

MQTT is an inter-device communication protocol. It was developed with the purpose of consuming little bandwidth using the publish / subscribe model. This model consists of defining two types of network entities: the message broker and clients. The broker acts as a server that receives the messages from the source clients and passes them to the target clients in push mode. The customer can be any device that can interact with the broker and can publish or subscribe to a topic. A topic is a data channel, where MQTT messages

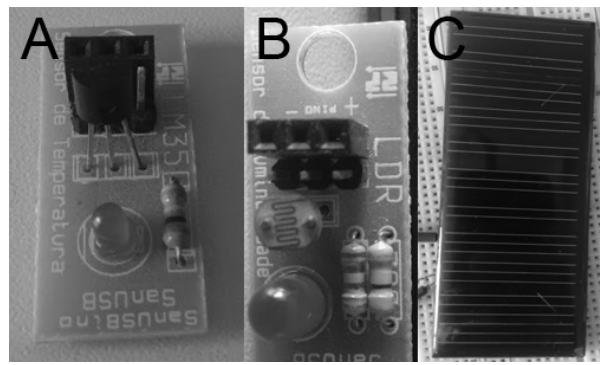

Fig. 2. Temperature sensor (A), LDR (B) and photovoltaic solar panel (C).

arrive to subscribing clients. An illustration of the communication flow of the MQTT communication protocol can be seen in Figure 3

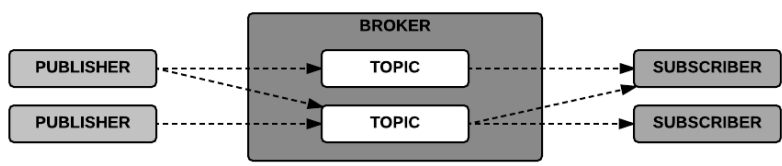

Fig. 3. Representation of the communication process and the participating entities in the MQTT protocol.

The MQTT protocol was implemented based on the TCP stack and therefore has a parameter for data reliability, that is, the guarantee that the message arrived at the destination, known as QoS (Quality of service), which assumes the values 0,1 or 2 according to the desired reliability level. This feature allows even low-quality networks (mobile or WiFi) to operate efficiently. As security measures, MQTT also offers the possibility of setting a password for the topic and using Secure Socket Layer (SSL) / Transport Layer Security (TSL) encryption.

\section{METHODOLOGY}

The purpose of this monitoring system is to collect data from the voltage generated by the solar photovoltaic module, the ambient temperature and the incidence of light, and send them to an MQTT topic of the broker located on the Federal Institute of Ceará (Brazil) server. On this server there are scripts that handle data and store the values that come to the topic in a database. Figure 4 shows a full representation of the proposed acquisition system.

\subsection{MQTT and data processing}

The embedded system sends the data via WiFi to the MQTT broker where there is a Python application capturing the messages and saving them in the database. A diagram showing the steps of the software running on the server can be seen in Figure 5

In the microcontroller the data is already sent in JSON format and each data type has a specific topic in the broker, so the program upon receiving them creates different tables for each type of data and then inserts the data.

\section{RESULTS}

Figure 6 shows the confirmation of data receipt on the broker and storage in the database. The developed codes are hosted in a public repository and can be found in [15].

On Figure 7 the proposed project mounted on protoboard can be seen. It is possible to see the microcontroller (A), connected to the 


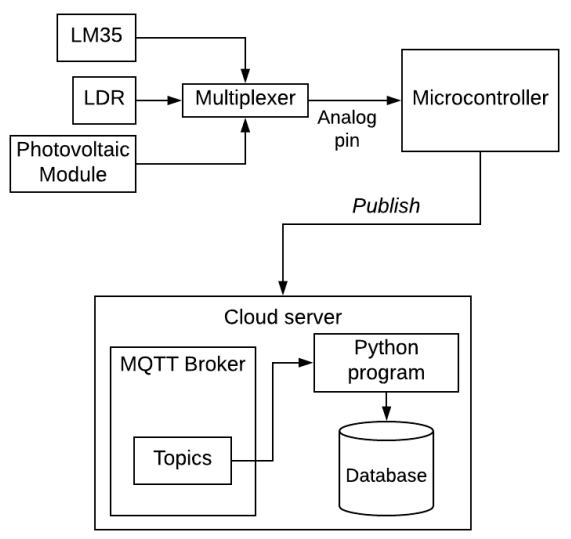

Fig. 4. Representation of the proposed monitoring system.

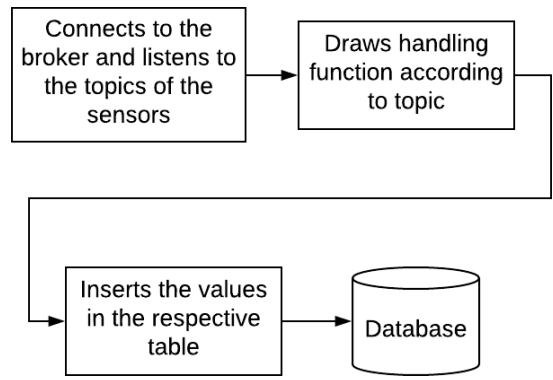

Fig. 5. Main steps of the python program.

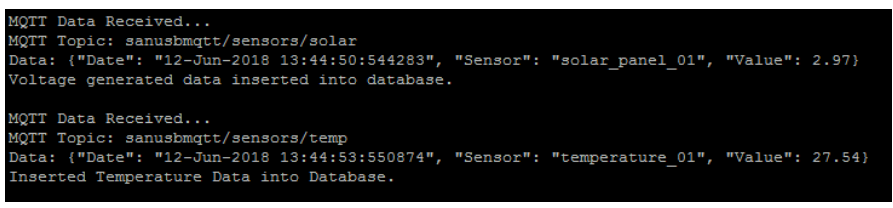

Fig. 6. Data received in the MQTT broker.

analog output from the multiplexer (B), which interprets three input values: photovoltaic module output voltage $(\mathrm{C})$, temperature sensor (D) and LDR (E); and sends to the microcontroller AD input pin.

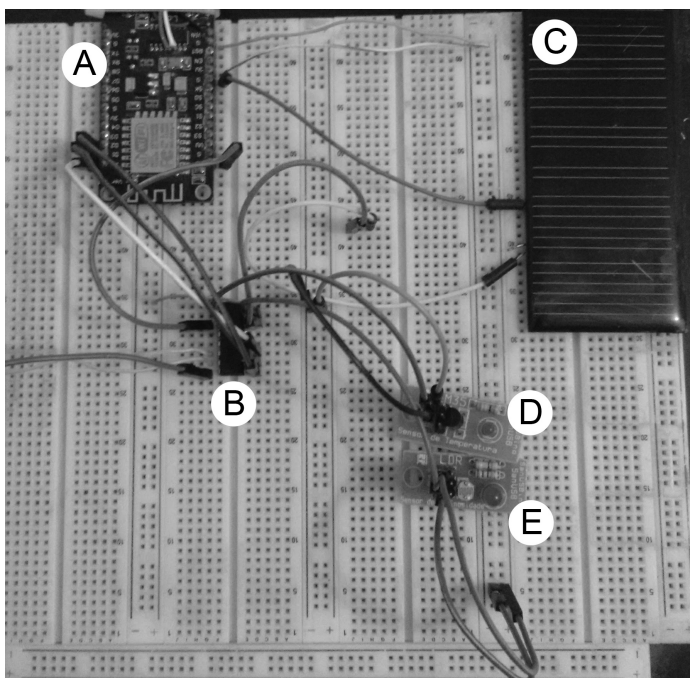

Fig. 7. Protoboard mounted project.

\section{FINAL CONSIDERATIONS}

Solar energy is a renewable source and is becoming popular in Brazil. Nowadays, Brazilians are looking for renewable energies as an alternative way of reducing residential spending. The monitoring system proposed in this work aims to monitor a solar photovoltaic module to indicate scenarios of greater power generation using sensors. The received data are stored in a cloud server database and can be visualized by any allowed remote user and a web page can be developed to display charts with the data stored. The MQTT topic can also be listened using any platform capable to connect to the MQTT broker.

Hereafter the project can be improved with data analysis using neural networks running on an embedded Linux system (such as Raspberry $\mathrm{Pi}$ ) to obtain a foresight of the measurements of the environment. This data predictions are important for fault analysis and to evaluate the production potential of a certain site electric energy relating to photovoltaic generation, which are influenced by meteorological information.

\section{REFERENCES}

[1] Rafael Amaral Shayani, Marco Aurélio Gonçalves de Oliveira, and Ivan Marques de Toledo Camargo. Comparison of the cost between solar photovoltaic and conventional sources. https://edisciplinas.usp.br/pluginfile. php/3427159/mod_resource/content/1/solar.pdf. 2006. [Online; accessed 30 may 2018].

[2] M. A. Green, K. Emery, Y. Hishikawa, W. Warta, and E. D. Dunlop. Progress in photovoltaics: research and applications. Wiley Online Library, v. 23, n. 1, p. 1-9, 2015., 2015.

[3] Renata I. S. Pereira, Paulo C. M. Carvalho, and Sandro C. S. Jucá. Wifi data acquisition system and online monitoring applied to thermoelectric microgeneration modules. Renewable Energy and Power Quality Journal (RE\&PQJ), (13):370373, 2015.

[4] Ramesh Nagappa Naik and Shruthi G. Article: Iot based secure smart home. IJCA Proceedings on National Conference on Electronics, Signals and Communication, NCESC 2017(4):7-10, July 2018. Full text available. 
[5] Renata I. S. Pereira, Ivonne M. Dupont, P. C. M. Carvalho, and Sandro C. S. Jucá. Iot embedded linux system based on raspberry pi applied to real-time cloud monitoring of a decentralized photovoltaic plant. Measurement, 114:286-297, 2018.

[6] Renata I. S. Pereira, Sandro C. S. Jucá, and Paulo C. M. de Carvalho. Online monitoring system for electrical microgeneration via embedded wifi modem. IEEE Latin America Transactions, 14(7):3124-3129, 2016.

[7] OASIS. Mqtt, message queuing telemetry transport. http: //mqtt.org/documentation 1999. [Online; accessed 30 may 2018].

[8] Zamir Chafekar, Mohd Husain Khan, Kuldeep Lakra, and S. B. Dhonde. Implementation of automatic gas accident prevention system using arduino and gsm. International Journal of Computer Applications, 180(47):5-7, Jun 2018.

[9] Everton Dornelas and Sérgio Campello Oliveira. Monitoramento de consumo doméstico de Água, utilizando uma metaplataforma de iot. http://revistas.poli.br/index. $\mathrm{php/repa/article/viewFile/575/187,} \mathrm{2017.} \mathrm{[Online;}$ accessed 30 may 2018]

[10] João Luis Grizinsky de Brito. A system for monitoring private, real-time and non-invasive energy consumption using arduino technology. http://www.uel.br/ctu/deel/TCC/ TCC2016_JoaoLuisGrizinskyBrito.pdf 2016. [Online; accessed 30 may 2018].

[11] Zatin Gupta, Tanay Sen, Vinayak Gupta, Vaishnavi, and Vijay. Home-automation using arduino-uno board and android app. International Journal of Computer Applications, 180(51):1820, Jun 2018.

[12] Thiago Henrique Deicke. Real-time monitoring system for urban acoustic levels based on wireless sensor networks. B.S. thesis, Universidade Tecnológica Federal do Paraná, 2016.

[13] NodeMCU. Nodemcu documentation. http://nodemcu. readthedocs.io/en/master/en/, 2017. [Online; accessed 30 may 2018].

[14] Texas Instruments. Cdx4hc405x, cdx4hct405x high-speed cmos logic analog multiplexers and demultiplexers. http:// www.ti.com/lit/ds/symlink/cd74hct4051.pdf 2017. [Online; accessed 30 may 2018].

[15] V. V. MOURA. Python program that manipulates and saves data coming from mqtt topics in database. https: //github.com/vitor-veras/mqtt-to-db 2018. [Online; accessed 12 june 2018]. 